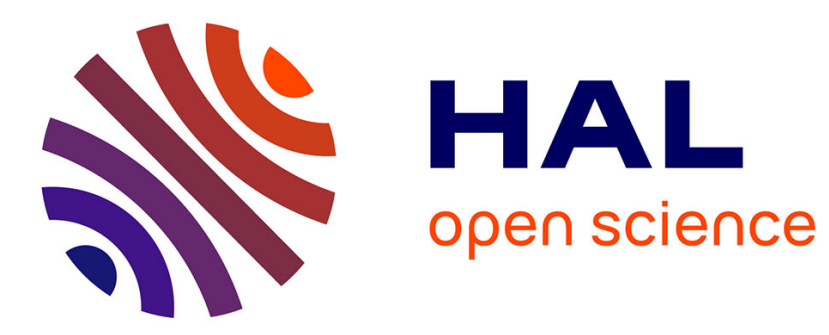

\title{
Convergent beam electron diffraction
}

D. Cherns

\section{- To cite this version:}

D. Cherns. Convergent beam electron diffraction. Journal de Physique IV Proceedings, 1993, 03 (C7), pp.C7-2113-C7-2122. 10.1051/jp4:19937336 . jpa-00251983

\section{HAL Id: jpa-00251983 https://hal.science/jpa-00251983}

Submitted on 1 Jan 1993

HAL is a multi-disciplinary open access archive for the deposit and dissemination of scientific research documents, whether they are published or not. The documents may come from teaching and research institutions in France or abroad, or from public or private research centers.
L'archive ouverte pluridisciplinaire HAL, est destinée au dépôt et à la diffusion de documents scientifiques de niveau recherche, publiés ou non, émanant des établissements d'enseignement et de recherche français ou étrangers, des laboratoires publics ou privés. 


\title{
Convergent beam electron diffraction
}

\author{
D. CHERNS
}

H.H. Wills Physics Laboratory, University of Bristol, Tyndall Avenue, Bristol BS8 1TL, U.K.

\begin{abstract}
The development of new materials requires an understanding of their mode of growth and the nature of defects, interfaces and strains thereby incorporated. This paper shows how convergent beam electron diffraction (CBED) and large angle CBED (LACBED) can be used to analyse such problems. It is shown how CBED and LACBED can give two-beam rocking curves, which can be used to profile plane rotations, strain and composition in multilayer structures, and to determine rigid body displacements at stacking faults and interfaces. In addition LACBED has become a powerful technique for the analysis of dislocations. The recent application of LACBED to determine the Burgers vectors of stair-rod and other partial dislocations is explained.
\end{abstract}

\section{Introduction}

CBED has been traditionally used to examine the structure of perfect crystals (e.g. see ref. [1]). However in the last few years it has become clear that CBED, and more particularly LACBED, provide powerful methods of analysing imperfect crystals. In this paper we concentrate on one particular development, namely the use of CBED and LACBED to generate "electron rocking curves". Firstly it is important to realise that the diffraction discs in convergent beam patterns show crystal rocking curve detail. As we cross a given diffraction disc the changing direction of the incident electron beam generates a scan of the diffraction function (fig 1). Since the diffraction angles are small $\left(\sim 1^{\circ}\right)$ the diffraction function is sampled in a direction approximately normal to $\mathbf{g}$ and thus contains information on the composition and strains as a function of depth in the crystal. If the incident beam is close to a prominent zone axis, the diffraction function for $g$ is usually dependent on many-beam interactions. This leads to the complex intensity variations across the discs in zone axis patterns which have been used to examine crystal symmetries. However, if the crystal is tilted appropriately we can achieve approximately two-beam conditions giving rocking curves that can be interpreted relatively simply.

In using electron rocking curves to analyse imperfect crystals, the LACBED technique (fig 2) is particularly useful. Since the specimen is not at beam crossover a selected area aperture can be used to remove all but one diffracted beam. This means that the convergence, and therefore the rocking, angle may be increased from the limit $\sim 1^{\circ}$ required in conventional CBED to avoid beam overlap to $5^{\circ}$ or more depending on the electron optics. It is also clear that the selected 


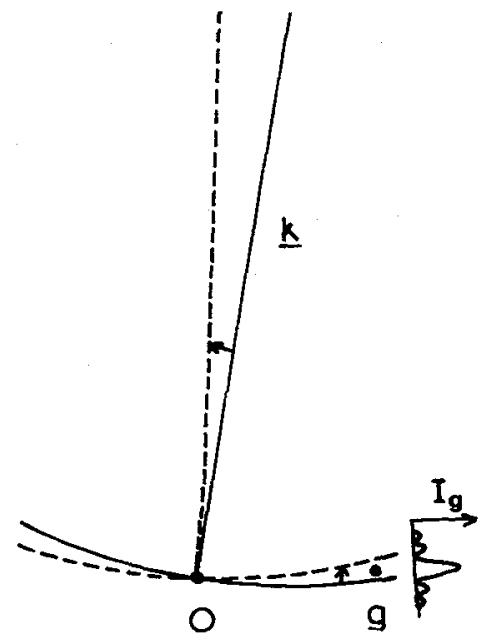

Fig 1. Changing the incident electron beam direction causes the Ewald sphere to sweep through the diffraction function for $\mathbf{g}$

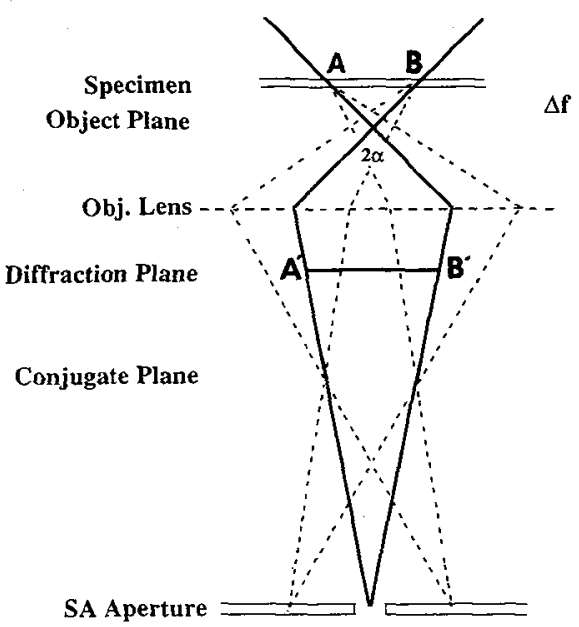

Fig 2. Schematic view of ray optics in the LACBED technique

area aperture can act as an efficient fitter of the thermal diffuse component of inelastic background [2]. This can give useful rocking curve information at angles of $10^{\circ}$ or more from the Bragg orientation, an important factor in studies of quantum well structures (see ref [3] for a review). The fact that a shadow image of the specimen appears in the LACBED disc (with a resolution given approximately by the minimum probe size) is important in allowing us to examine the changes in rocking curve detail on approaching defects or interfaces. This has proved especially useful in applying convergent beam techniques to the study of dislocations (see later).

\section{Strains in epitaxial layers}

LACBED has proved very effective for profiling strains in epitaxial layers. In a cubic system in (001) orientation, layer strains lead to a tetragonal distortion. This is equivalent to a relative rotation of crystal planes between adjacent layers given approximately by

$$
\delta \theta=\varepsilon / 2 \frac{(1+v)}{(1-v)} \sin 2 \theta
$$

where $\varepsilon$ is the relative strain and $v$ is Poisson's ratio $[4,5]$. Kiely and Cherns [4] used LACBED to profile the resulting splitting of the diffraction spots in $\mathrm{NiSi}_{2} /(001) \mathrm{Si}$ bicrystals $(\varepsilon=0.004)$ using plan-view samples. However the LACBED technique has also been applied to strained layers prepared in cross-section. Fig 3 illustrates some recent work on Si/SiGe multilayers. LACBED is insensitive to the layer strains when the cross-section is edge-on since the spot splitting is in the horizontal plane i.e approximately tangential to the Ewald sphere. However as we tilt away from the horizontal about the $[1 \overline{1} 0]$ axis (left to right) strain becomes visible as a 

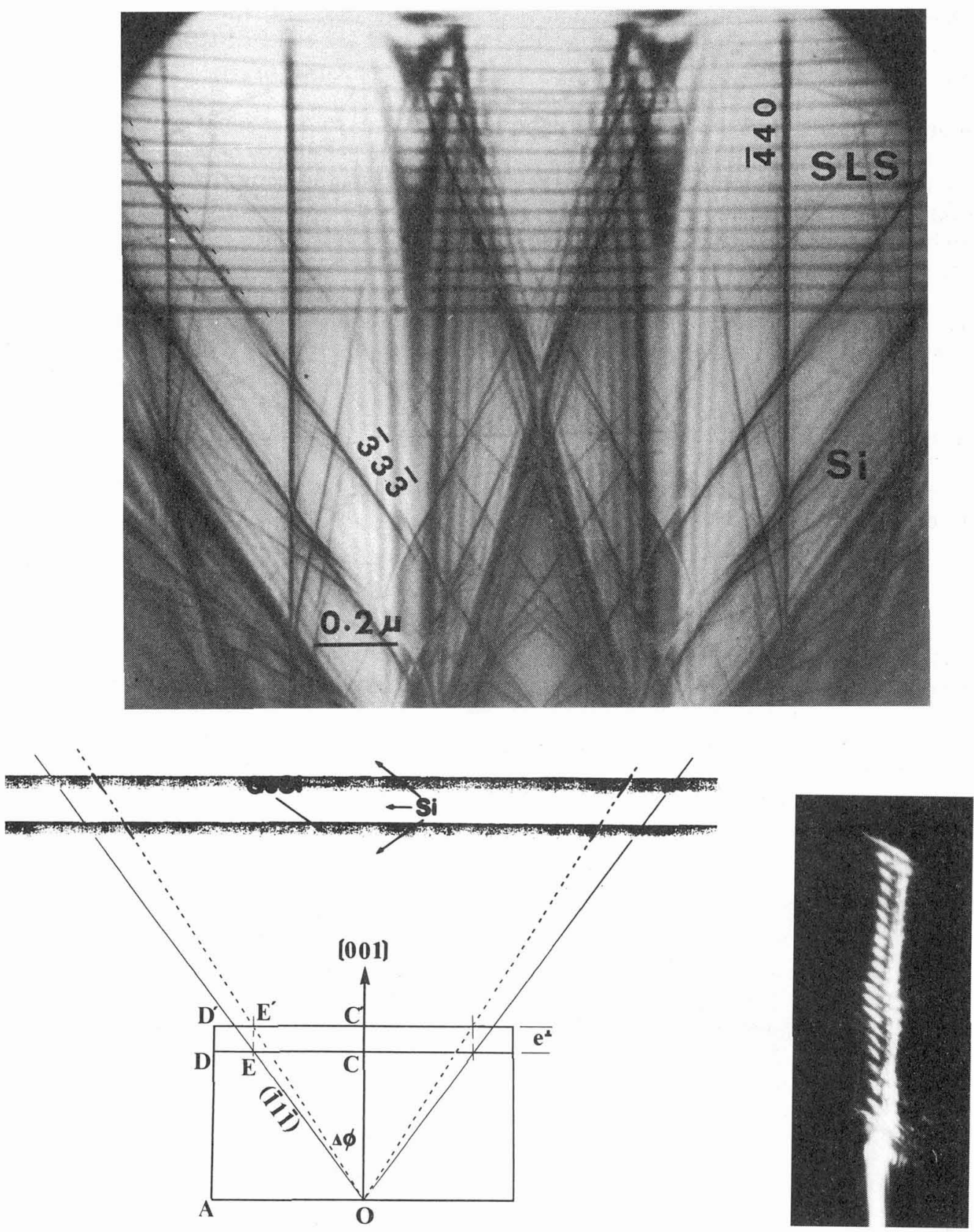

Fig 3. Top: bright field LACBED pattern from a $\mathrm{Ge}_{0.2} \mathrm{Si}_{0.8}(100 \AA) / \mathrm{Si}(400 \AA)$ superlattice on $\mathrm{Si}$ showing displacements of $\{333\}$ contours crossing the superlattice. The displacements have been indicated on the $\overline{3} 3 \overline{3}$ contour [courtesy of XF Duan]. Bottom left: a schematic illustration of the displacements of 333 contours. Bottom right: a darkfield LACBED pattern from a $\mathrm{Ge}_{0.33}(200 \AA) / \mathrm{Si}(450 \AA)$ superlattice using a 333 reflection shows the displacements more clearly [courtesy of $Y$ Atici]. 
progressive displacement of diffraction contours such as $\overline{3} 3 \overline{3}$ on passing from the $\mathrm{Si}$ to the $\mathrm{SiGe}$ layers. The effect can be observed both in bright field or dark field LACBED patterns as illustrated.

A quantitative analysis of the layer strains is complicated in the cross-sectional geometry by the fact that interfaces must be tilted from edge-on such that adjacent layers overlap, and by thin foil relaxation. The relaxation occurs along the specimen normal leading to an orthorhombic rather than a tetragonal distortion. It can, however, be separated from the true crystal distortion by comparing LACBED patterns from different reflections and a procedure to do this has recently been described by Duan et al [6]. Nevertheless the analysis of strain is perhaps better carried out using plan-view samples since this essentially avoids foil relaxation problems. Despite the fact that information from the constituent layers is now superimposed, average layer strains in plan-view multilayer samples may be easily measured to $\sim 20 \%$ accuracy [7].

\section{Displacements at stacking faults and interfaces}

We can get useful insight into electron rocking curves by using the kinematical expression for the scattered amplitude given by

$$
\phi_{g}=\int_{0}^{t} F(z) e^{-2 \pi i(s z+\underline{g} \cdot \underline{R}(z))} d z
$$

where $F(z)$ is the structure factor at depth $z, s$ is the deviation parameter and $R(z)$ is any crystal displacement. Layer strains are represented to first order by changes in deviation parameter. For a stacking fault or interface with a rigid body displacement $\mathbf{R}$, the rocking curve generally becomes asymmetric. Morniroli and Steeds [8] have shown how this asymmetry can be used as a simple method of determining the type of stacking faults in f.c.c. structures. By comparing theoretical and experimental rocking curves Al-Khafaji et al $[9,10]$ have shown how LACBED can be used to analyse interface displacements. Fig 4 illustrates how LACBED was used to analyse the structure of the $\mathrm{CoSi}_{2} /(111) \mathrm{Si}$ interface.

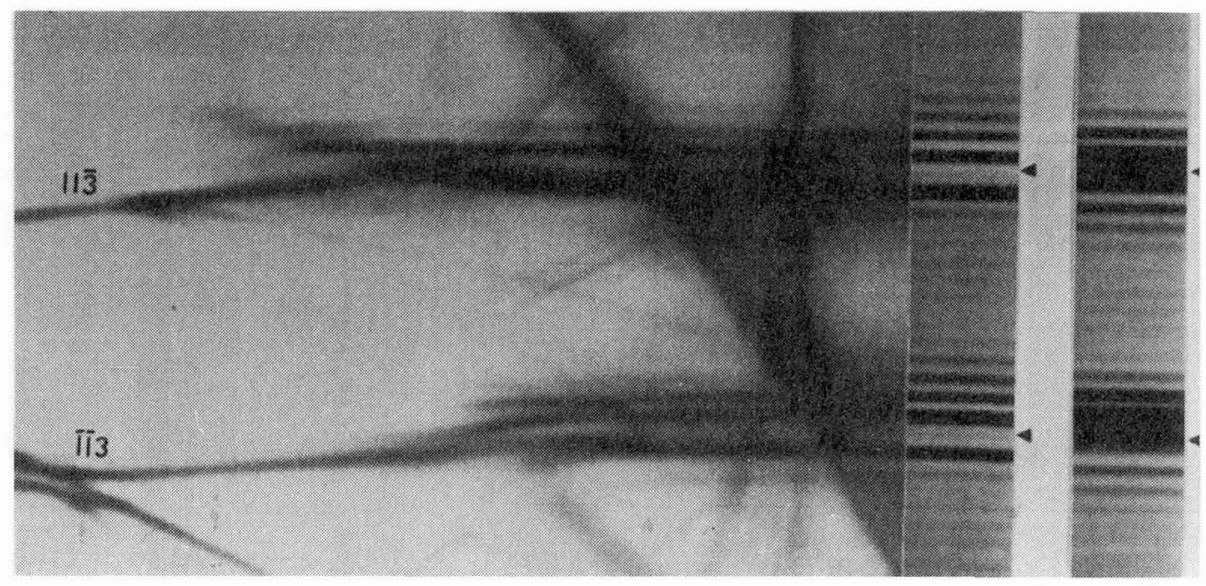

Fig 4. Left: experimental bright field LACBED pattern from a $\mathrm{CoSi}_{2} /$ (III)Si bicrystal showing 311 contours. Right: computations for the "7-fold" (left) and "5-fold" (right) models of $\mathrm{CoSi}_{2} /(111) \mathrm{Si}$ [see ref [9] for further details] 


\section{Periodic multilayers}

For plan-view multilayer structures which are periodic, equation (2) predicts that rocking curves should display superlattice reflections. LACBED studies of the composition profile have been carried out using superlattice reflections from periodic AlGaAs/GaAs, InP/InGaAs, $\mathrm{CdMnTe} / \mathrm{CdTe}$ and $\mathrm{Si} / \mathrm{SiGe}$ structures and the reader is referred to references $\{3,7,11]$ for more details. Using "composition-sensitive" reflections up to nearly 20 orders of superlattice reflections have been observed [11]. In this respect electron rocking curves compare well with the equivalent $X$-ray rocking curve method since the spatial resolution is much better, as low as $10-20 \AA$ in recent work (e.g. see ref [6]). This means that we can largely avoid spatial averaging due to factors such as interface roughness and sample bending which act to attenuate higher order satellites. LACBED patterns from period multilayers can give the period to monolayer precision and have been used to identify spatial variations in the period and constituent layer thicknesses $[3,12]$. The separation of strain and composition profiles is possible since we can choose reflections from planes parallel or perpendicular to the growth direction which are insensitive to layer strains (equation (1)) or reflections from inclined planes where strain becomes a factor.

\section{Single buried layers}

Considerable progress has been made recently in analysing electron rocking curves from single quantum well (SQW) structures which consist of a single buried layer surrounded by much thicker cladding or barrier layers [13-15]. A typical LACBED for an InP/InGaAs SQW structure is shown in fig 5 . Basically the pattern can be interpreted as a single crystal rocking

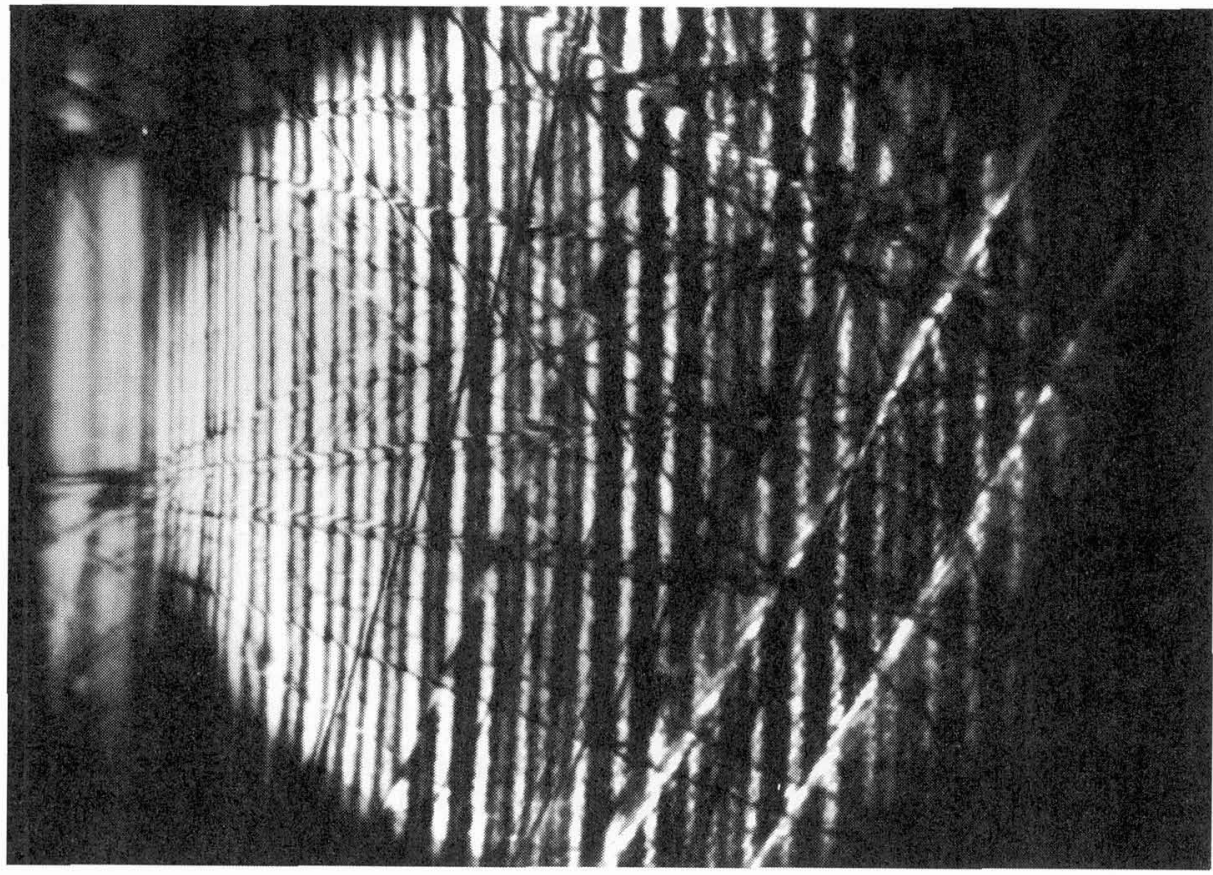

Fig 5. A 200 dark field LACBED pattern from a $1050 \AA$ InP/ $20 \AA$ In 0.53 Ga $0.47 A s / 1150 \AA$ InP sample over the range $0<\mathrm{s}<3 \times 10^{-2} \AA^{-1}$ [Courtesy of I K Jordan] 


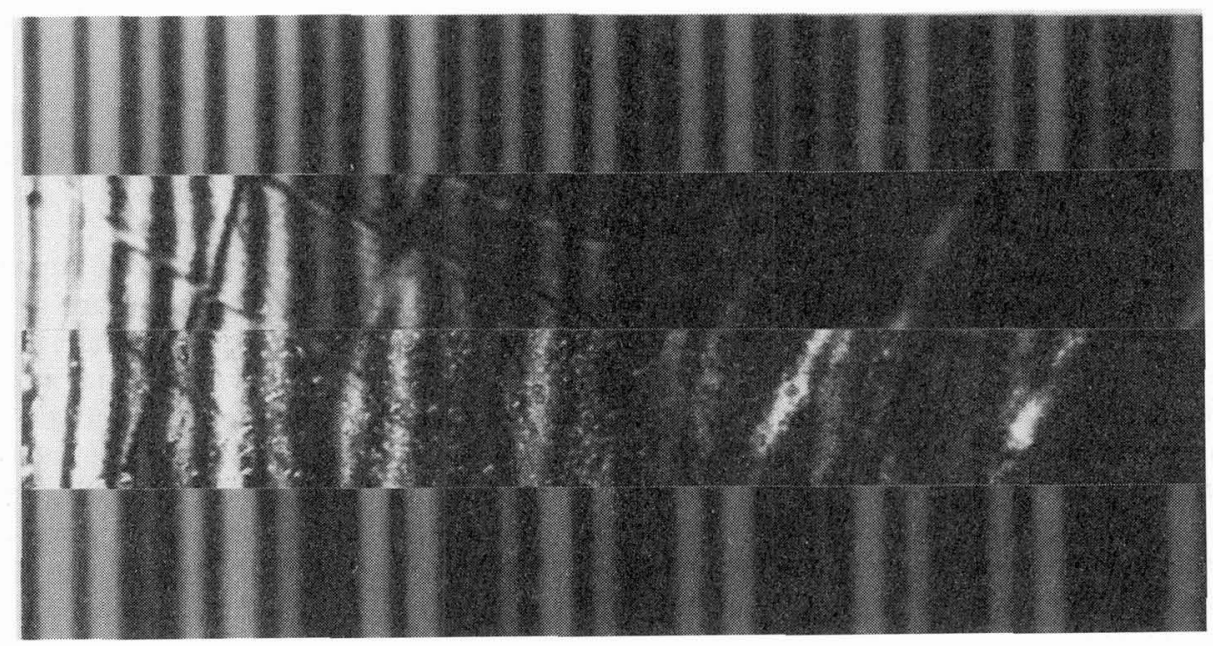

Fig 6. Details of 200 rocking curves for $\mathrm{In}_{0.53} \mathrm{Ga}_{0.47}$ As samples with ultra-thin P-substituted layers. Top and bottom theoretical curves assume $P$ layers one and two monolayers thick respectively $\left(0.004<\mathrm{s}<0.014 \AA^{-1}, \mathrm{~s}\right.$ increasing left to right) and are in reasonable agreement with the adjacent experimental results. [Courtesy of $N$ Grigorieff, see ref [15] for more details]

curve with subsidiary maxima whose intensities decay monotonically with increasing s which is itself modulated by the presence of the buried $20 \AA$ InGaAs quantum well. The amplitude of the modulation depends both on the quantum well thickness and its depth in the foil and a simple kinematical analysis suffices to measure the local well thickness to about a monolayer. The technique has recently been applied to detect ultra thin buried layers [15] and to locate individual interface atomic steps $[3,14,15]$. As an example of the former, fig 6 shows 200 LACBED patterns from two InGaAs samples grown by MOCVD which were subjected to a purge of phosphine gas during a short growth interrupt. This procedure leads to near-atomic phosphorus-rich layers whose thickness depends on the growth interrupt time. In fig 6 we see two theoretical rocking curves assuming complete substitution of one and two arsenic layers by phosphorus (top and bottom respectively). The increase in the modulation on passing from one to two substituted layers is clearly visible, particularly in the weaker fringes. The experimental rocking curves represent different exposure times to phosphine and superficially appear to agree quite well in each case with the adjacent theoretical profiles. This has been confirmed by a more quantitative analysis and the reader is referred to the paper by Grigorieff et al [15] for further details.

\section{Rotations across grain boundaries}

Both CBED and LACBED can be applied to measure rotations across grain boundaries. Fig 7 shows an interesting example where LACBED has been used to profile plane rotations across 
near edge-on twin boundaries in $\mathrm{YBa}_{2} \mathrm{Cu}_{3} \mathrm{O}_{7-\mathrm{y}}$. The geometry is, in fact, very closely similar to that illustrated in fig 3 , the plane rotation being, in this case, $\sim 1^{\circ}$. The plane rotation is a function of the oxygen content and patterns such as those in fig 7 have been used to analyse variability in the oxygen content and changes brought on by electron irradiation. More details are given in the paper by Midgley et al [16].

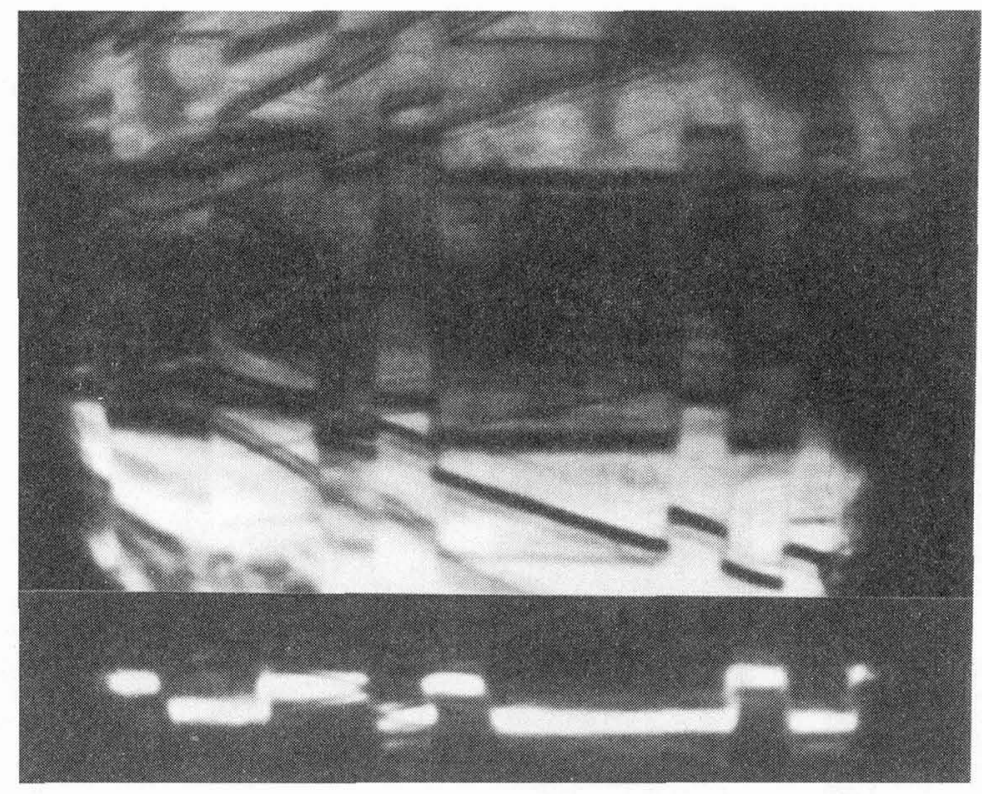

Fig 7. Bright and dark field LACBED patterns from a $\mathrm{YBa}_{2} \mathrm{Cu}_{3} \mathrm{O}_{7}-\mathrm{y}$ sample with twins. The sample is tilted so that the $\{110\}$ twin boundaries are a few degrees away from edge-on. The contour displacements, which indicate plane rotations $\sim 1^{\circ}$, increase as the twin boundaries are tilted from the exact edge-on orientation reflecting a geometry close to that illustrated in fig 3

\section{Dislocations}

[Courtesy of P A Midgley, for more details see ref [16]].

The application of LACBED to the study of dislocations has been particularly effective. It was shown by Cherns and Preston $[17,18]$ that relatively high order diffraction contours dissociate on crossing dislocations (e.g. see fig 8 ) giving a number of subsidiary maxima, $n$, given by the rule

$$
|\mathbf{g} \cdot \mathbf{b}|=\mathrm{n}+1
$$

Moreover the direction in which the contour dissociates depends on the sign of the Burgers vector. This has provided a very attractive method of analysing dislocation Burgers vectors since both the magnitude of $\mathbf{b}$ and its sign are, in general, difficult to establish by imaging 
methods. The characteristics of LACBED patterns from dislocations are dependent principally on topology and are relatively insensitive to factors such as dislocation depth and foil thickness. Breakdowns of the rule have been observed where the dislocation is very close to one of the foil surfaces, where very strongly dynamical conditions exist [19] and in materials such as $\beta$-brass which are highly anisotropic (J-P Morniroli - private communication).

The fact that LACBED allows us to investigate contours with large g-vectors suggests that the technique should allow us to examine dislocations with small Burgers vectors i.e. the magnitude of $|\mathbf{g} \cdot \mathbf{b}|$ can be thereby increased. Recent work by Morniroli and Cherns [20] has shown that the technique may be successfully applied to study a range of partial dislocations. For simplicity it is important to choose reflections in which adjoining stacking faults are out of contrast. Fig 8 shows a partial dislocation terminating a stacking fault in a CdMnTe/CdTe bicrystal using a reflection of $\left\{\begin{array}{ll}102 & 2\end{array}\right\}$ type in which $\mathbf{g} \cdot \mathbf{R}=$ integer where $\mathbf{R}$ is the fault vector. We see a LACBED pattern which immediately suggests a value of $\mid$ g.b $\mid=4$ (i.e. $n=3$ ) in accord with a Burgers vector of Frank type $b=\frac{1}{3}[1 \overline{1} 1]$.
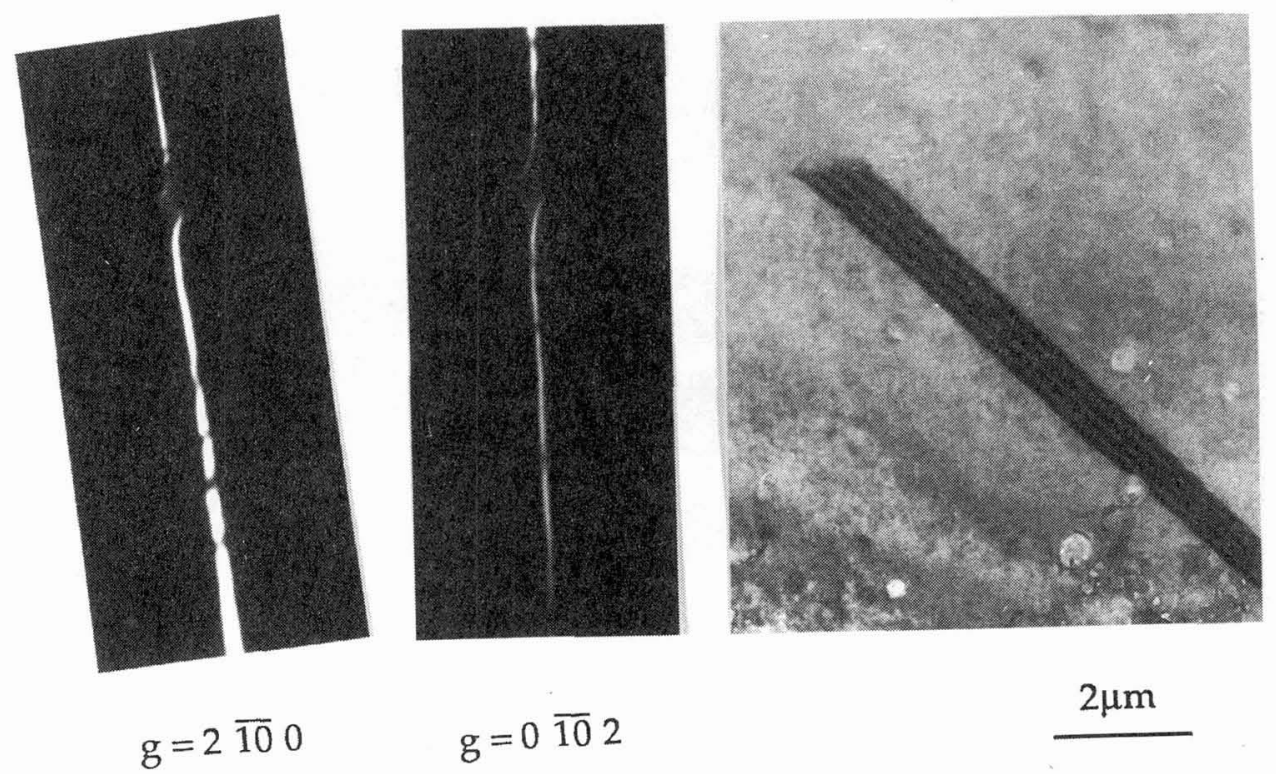

Fig 8. Interaction of $\left(\begin{array}{ll}10 & 2\end{array}\right)$ contours with a partial dislocation in a CdMnTe/CdTe bicrystal. The partial is believed to be of Frank type $b=\frac{1}{3}[1 \overline{1} 1]$.

Methods for the general analysis of Shockley and Frank dislocations in f.c.c.-related structures are outlined in a forthcoming publication [22]. In fact reflections of type $\left\{\begin{array}{lll}10 & 2 & 0\end{array}\right\}$ are particularly useful since different values of $\mathbf{g} \cdot \mathbf{b}$ are obtained for the four possible partial Burgers vectors ( 1 Frank and 3 Shockley) which can terminate a single stacking fault. Thus an analysis is, in principle, possible using only this one reflection. The general method has also 
been extended to examine the smaller partial dislocations present at stacking fault intersections (stair-rod dislocations). Fig 9 shows a good example where a stair-rod dislocation at intersecting faults in a stacking fault pyramid in $S i$ is intersected by a reflection of $\left\{\begin{array}{ll}10 & 20\end{array}\right\}$ type. The intersection shows a $|\mathbf{g} \cdot \mathbf{b}|=2$ image consistent with a Burgers vector of $\frac{1}{6}\langle 110\rangle$ type, a result confirmed by a more general LACBED analysis [22].
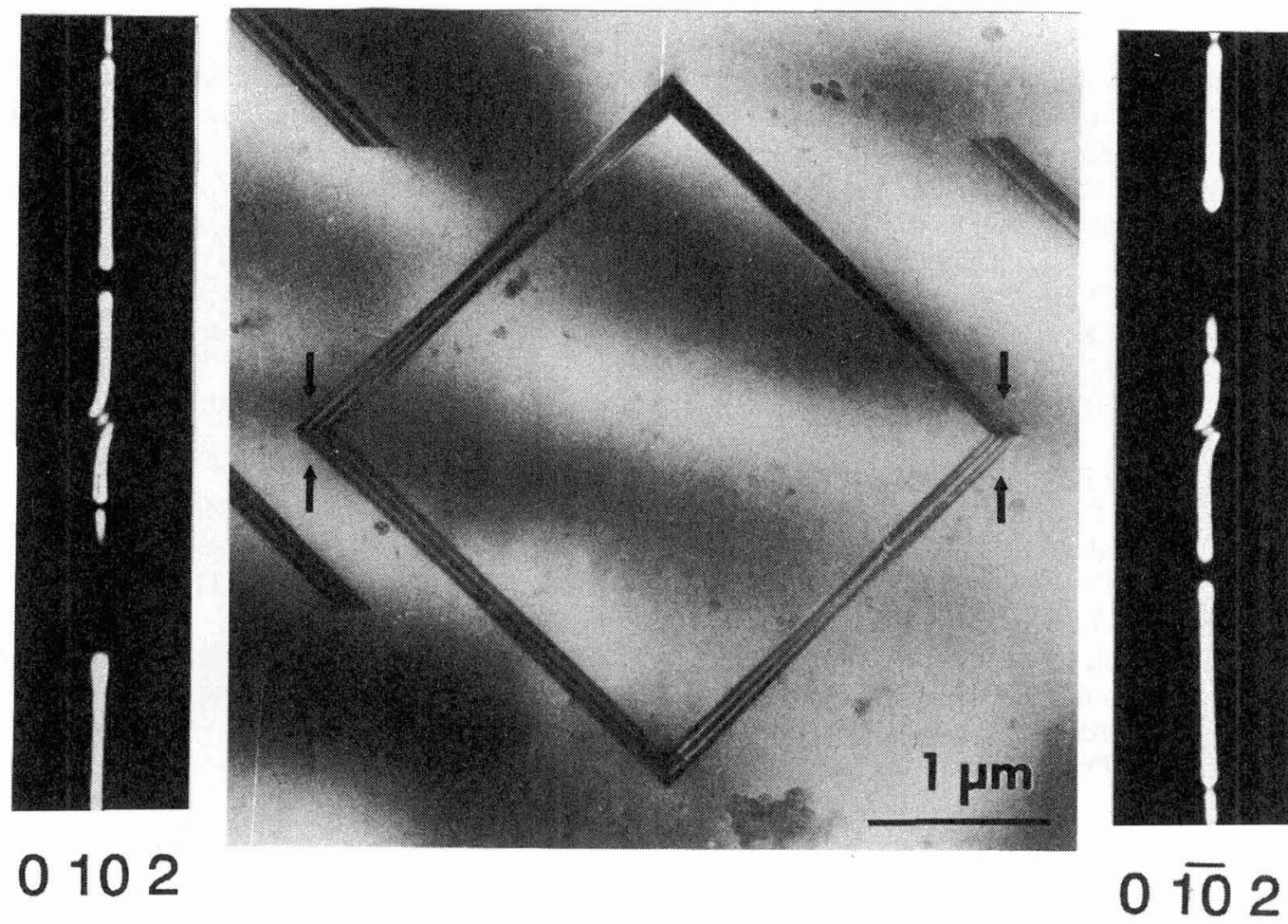

Fig 9. Part of an analysis of stair-rod dislocations present at intersections between faults in a stacking fault pyramid in $\mathrm{Si}$. The stair-rods are believed to have Burgers vectors

$$
b=\frac{1}{6}\langle 110\rangle \text { type. }
$$

The application of LACBED to determine small Burgers vectors suggests that, in the future, we might use LACBED to examine dislocations in grain boundaries. At present the main limitation of the technique appears to be that we need to examine detail in the LACBED patterns close to the Bragg position $(s=0)$. The spatial resolution of the technique is thus limited by the typical dislocation image width of a few hundred $\AA$ suggesting that the fine structure of dissociated dislocations or dense interfacial network of dislocations will not be accessible to study by LACBED. 


\section{References}

[1] Steeds J.W., "Review of convergent beam electron diffraction", Societa Italiana Microscopia Elettronica, Rome (1986) 219-280.

[2] Jordan I.K., Rossouw C.J. and Vincent R., Ultramicroscopy 35 (1991) 237.

[3] Cherns D., Inst. Phys. Conf. Ser. 117 (1991) 549.

[4] Kiely C.J. and Cherns D., Inst. Phys. Conf. Ser. 76 (1985) 183.

[5] Cherns D., Kiely C.J. and Preston A.R., Ultramicroscopy 24 (1988) 355.

[6] Duan X.F., Grigorieff N., Cherns D., Steeds J.W. and Sheng C., Int. Conf. on Microscopy of Semiconducting Materials, Oxford, April 1993: Proceedings in press.

[7] Cherns D., Touaitia R., Preston A.R., Rossouw C.J. and Houghton D.C., Phil. Mag. A64 (1991) 597.

[8]. Morniroli J-P. and Steeds J.W., Inst. Phys. Conf. Ser. 119 (1991) 417.

[9] Al-Khafaji M.A., Cherns D., Rossouw C.J. and Hull R., Inst. Phys. Conf. Ser. 119 (1991) 51.

[10]. Al-Khafaji M.A., Cherns D., Rossouw C.J. and Woolf D.A., Inst. Phys. Conf. Ser. 117 (1991) 253.

[11] Vincent R., Wang J., Cherns D, Bailey S.J, Preston A.R. and Steeds J.W., Inst. Phys. Conf. Ser. 90 (1987) 233.

[12] Jordan I.K., PhD Thesis (1991) University of Bristol.

[13] Cherns D., Jordan I.K. and Vincent R., Phil. Mag. Lett. 58 (1988) 45.

[14] Jordan I.K., Cherns D., Hockly M. and Spurdens P., Inst. Phys. Conf. Ser. 100 (1989) 293; Proc. of 12 Int. Congress on Electron Microscopy, Seattle 1990, Vol 4, 680.

[15] Grigorieff N., Cherns D., Yates M.J., Hockly M., Perrin S.D. and Aylett M.R., Phil. Mag: in press.

[16] Midgley P.A., Vincent R. and Cherns D., Phil. Mag. A66 (1992) 237.

[17] Cherns D. and Preston A.R., Proc. of 11th Int. Congress on Electron Microscopy, Kyoto (1986), ed. Imura T. et al, Vol 1, 721.

[18] Cherns D. and Preston A.R., J. Electron Microscope Technique, 13 (1989) 111.

[19] Chou C.T., Preston A.R. and Steeds J.W., Phil. Mag. A65 (1992) 863.

[20] Morniroli J-P. and Cherns D., "The analysis of dislocations with small Burgers vectors by convergent beam electron diffraction". Proceedings of 32 nd annual meeting Société Française de Microscopie Electronique, Rouen, France, June 1992.

[21] Cherns D. and Morniroli J-P.: manuscript in preparation. 